\title{
Compte rendu de livre
}

\section{James C. Scott. Homo Domesticus. Une histoire profonde des premiers États. Paris: La découverte, 2019, 301 pages.}

Jean Michaud, Université Laval

allait-il s'attendre à ce que, au tournant de ses 80 ans, un James C. Scott
repentant délaisse la plus vive passion de sa carrière intellectuelle: la critique radicale de l'état? Évidemment, non. En revanche, on pourrait trouver déroutant que ce politologue aux pratiques souvent anthropologiques s'aventure aussi profondément sur le terrain de l'Histoire, qu'il avait déjà arpenté sur quelques siècles dans son ouvrage de 2009, The Art of Not Being Governed. An Anarchist History of Upland Southeast Asia. ${ }^{\mathrm{I}}$ Les historiens l'avaient écorché au passage sur ses méthodes inorthodoxes. Mais plusieurs avaient également salué la pertinence de son projet. Et son lectorat semble lui donner raison : à ce jour, l'ouvrage a été cité à plus de 5300 reprises, dépassant largement en 12 ans le canonique Political Systems of Highland Burma d'Edmund Leach portant sur les mêmes populations, mais publié 50 ans auparavant. Scott a ses défauts, mais il ne laisse pas indifférent.

Et le moins qu'on puisse dire concernant son dernier opus, c'est qu'il n'a pas froid aux yeux non plus. Dans Homo Domesticus, ${ }^{2}$ il rempile dans la relecture critique de l'Histoire officielle, cette fois étendue sur dix millénaires, en entendant faire vaciller sur son piédestal le mythe de la Révolution néolithique. Rien de moins.

En sept chapitres d'une efficacité redoutable et d'une incontestable érudition, Scott déconstruit pierre par pierre le savoir reçu sur la naissance 'révolutionnaire' de l'état au néolithique pour insister plutôt sur la construction structurelle des inégalités sociales. Adoptant délibérément le point de vue du paysan, il souligne les paradoxes du grand récit de la naissance de l'état et de la 'civilisation', explique dans des termes critiques la domestication du feu, des plantes, des animaux et de nous-mêmes, sapant vigoureusement l'exposé convenu des conséquences positives de l'agriculture et insistant, avec force 
exemples concrets, sur notre autodomestication, là où on attendait un éloge des prouesses technologiques d'une humanité en mouvement. Il souligne le fait que les céréales (la culture sédentarisée par excellence), les murailles (le retranchement des élites dans leurs chateaux-forts) et l'écriture (un véhicule par lequel les élites surveillent et définissent la culture) ${ }^{3}$ sont au cœur du projet pluri-millénaire de mise au pas des populations par la domestication, la servitude et la guerre. Ainsi créés, les espaces autoproclamés de civilisation fournissent l'aulne à laquelle se mesure en creux la barbarie qui afflige tous ceux qui ne sont pas 'nous' ou 'comme nous.'

Le chapitre 3 à lui seul vaut la lecture. Intitulé «Zoonoses: la tempête épidémiologique parfaite», sa description critique de la mise en place des conditions propices aux grandes pandémies apparait aujourd'hui prémonitoire.

Un nouveau livre de James C. Scott est toujours largement commenté dans les milieux académiques, mais il est aussi traité comme événement littéraire aux États-Unis et dans le monde anglophone. Pour qui en douterait, Against the Grain a ainsi été commenté dans The New York Times, The New York Review of Books, The London Review of Books, The New Yorker, The Financial Times, et The Wall Street Journal entre autres.

Fruit d'une recherche méticuleuse et détaillée, on a ainsi dit de sa thèse qu'elle constituait un réquisitoire, la réaction sceptique d'un outsider face aux idées reçues sur l'évolution des systèmes agricoles et les premiers états de Mésopotamie. A l'appui, la somme des références sur un vaste horizon intellectuel épate. Barry Cunliffe (European Archaeology, Oxford) écrit dans l'influent The Guardian : «Scott's original book is history as it should be written.»

Malgré tout, comme ce fut le cas pour son livre de 2009, on peut ici aussi reprocher à Scott une connaissance partielle et surtout, partiale de l'histoire sur la longue durée, et une utilisation des sources déterminée avant tout par le désir de soutenir son argument 'contre l'état.' Il est manifeste que Scott ne recule pas devant la polémique, quitte à s'improviser historien ou anthropologue au besoin.

Faut-il s'en offusquer? A sa décharge, ici encore Scott voit plus loin que les questions de méthode et de plates-bandes disciplinaires. Il entend ausculter, oui, mais uniquement dans un registre lui permettant de démontrer la justesse de ses intuitions, qui demeurent son véritable guide - et j'ajouterais: l'une de ses plus grandes forces. Il improvise, donc, et par moments s'improvise, mais le fil directeur de son projet depuis 40 ans, ce livre en étant l'héritier, est de parler en faveur des petits, des laissés pour compte, de celles et ceux qui subissent le 
pouvoir d'en haut-serfs, esclaves, paysans, dominés - sans avoir les moyens politiques ou militaires de le contredire ou de lui tenir tête. En ce sens, Homo Domesticus remplit ses promesses de continuité dans l'œuvre inspirante d'un maitre de l'écriture dépouillée et de la communication inclusive, conditions dans son esprit pour contribuer activement à l'affranchissement des masses tenues à la marge par l'élitisme politique autant qu'intellectuel, et par le pouvoir obstinément inégalitaire. La lecture de James C. Scott émancipe.

\section{Notes}

- Traduit et publié en 2013 au Seuil sous le titre accrocheur et tronqué de Zomia ou l'Art de ne pas être gouverné.

2 Against the Grain. A Deep History of the Earliest States, paru en 2017. Contrairement à l'ouvrage de 2009, il faut saluer le fait que les éditions La Découverte aient fait l'effort de laisser au livre son sous-titre original. Mais ce faisant, ils loupent inexplicablement l'occasion de rendre un hommage mérité à la brillante formule Against the Grain, qui aurait si bellement pu donner Contre le grain et conserver son double sens. Mais peut-être les éditeurs parisiens ne pratiquent-ils pas beaucoup la menuiserie.

3 Dans The Art of Not Being Governed, Scott avait déjà consacré un chapitre entier (numéroté $61 / 2$ ) au refus de l'écriture comme tactique de liberté par des populations désireuses de rester loin des griffes de l'état prédateur, une proposition polémique s'il en est (Michaud 20I7, 2020).

\section{Références}

Cunliffe, Barry, 20I7. «Against the Grain by James C. Scott review - the beginning of elites, tax, slavery » The Guardian, Saturday, November 25.

Michaud, Jean, 20I7. «What's (Written) History For? On James C. Scott's Zomia, Especially Chapter 61/2». Anthropology Today, 33 (I): 6-Io. https://doi.org/IO.IIII/I467-8322.I2322.

Michaud, Jean, 2020. «The Art of Not Being Scripted So Much. The Politics of Writing Hmong Language(s)». Current Anthropology, 6I (2): 240-63. https://doi. org/IO.IO86/708I43.

Scott, James C., 2009. The Art of Not Being Governed. An Anarchist History of Upland Southeast Asia. New Haven, Yale University Press. 